\title{
Testing the scalar sector of the twin Higgs model at colliders
}

\author{
Zackaria Chacko, ${ }^{1,2}$ Can Kilic, ${ }^{3}$ Saereh Najjari, ${ }^{4}$ and Christopher B. Verhaaren ${ }^{5}$ \\ ${ }^{1}$ Maryland Center for Fundamental Physics, Department of Physics, University of Maryland, \\ College Park, Maryland 20742-4111, USA \\ ${ }^{2}$ Theoretical Physics Department, Fermilab, P.O. Box 500, Batavia, Illinois 60510, USA \\ ${ }^{3}$ Theory Group, Department of Physics and Texas Cosmology Center, University of Texas at Austin, \\ Austin, Texas 78712, USA \\ ${ }^{4}$ Faculty of Physics, University of Warsaw, Pasteura 5, 02-093 Warsaw, Poland \\ ${ }^{5}$ Center for Quantum Mathematics and Physics (QMAP), Department of Physics, University of California, \\ Davis, California 95616-5270, USA
}

(Received 7 December 2017; published 22 March 2018)

\begin{abstract}
We consider mirror twin Higgs models in which the breaking of the global symmetry is realized linearly. In this scenario, the radial mode in the Higgs potential is present in the spectrum and constitutes a second portal between the twin and SM sectors. We show that a study of the properties of this particle at colliders, when combined with precision measurements of the light Higgs, can be used to overdetermine the form of the scalar potential, thereby confirming that it possesses an enhanced global symmetry as dictated by the twin Higgs mechanism. We find that, although the reach of the LHC for this state is limited, future linear colliders will be able to explore a significant part of the preferred parameter space, allowing the possibility of directly testing the twin Higgs framework.
\end{abstract}

DOI: 10.1103/PhysRevD.97.055031

\section{INTRODUCTION}

The discovery of the Higgs boson at CERN [1,2], while completing the standard model (SM), has also brought the hierarchy problem, the question of the radiative stability of the Higgs mass, into sharper focus. Symmetry-based solutions to the hierarchy problem [3-5] require new particles with masses at or below the $\mathrm{TeV}$ scale that have sizable couplings to the Higgs. Searches for these particles continue to be a major focus of the Large Hadron Collider (LHC) program. These analyses have become increasingly powerful and sophisticated in an effort to explore all simple realizations of the known cancellation mechanisms. So far, these searches, while imposing stiff constraints on solutions to the hierarchy problem, have provided no hints as to its resolution.

One explanation of these null results is that while the Higgs mass is indeed protected by a symmetry, the new particles associated with this symmetry are not charged under SM color. These states are then much more difficult to produce at a hadron collider, which complicates efforts to discover them. Several theories of this type have been

Published by the American Physical Society under the terms of the Creative Commons Attribution 4.0 International license. Further distribution of this work must maintain attribution to the author(s) and the published article's title, journal citation, and DOI. Funded by SCOAP ${ }^{3}$. proposed that stabilize the Higgs mass up to scales of order 5-10 TeV, the precision electroweak scale [6-14]. The best-known example of this class of models is the mirror twin Higgs (MTH) [6], in which the symmetry partners are neutral, not just under SM color, but under all the SM gauge groups.

In the MTH framework, the particle content of the SM is extended to include a mirror ("twin") copy of all the fields in the SM. A discrete $Z_{2}$ twin symmetry relates the particles and interactions in the SM and mirror sectors. The Higgs sector respects a larger global symmetry which, in the simplest incarnation of the model, is taken to be $\mathrm{SU}(4) \times \mathrm{U}(1)$. This global symmetry, like the discrete symmetry, is only approximate. The electroweak gauge symmetries of the SM and twin sectors are embedded inside the global symmetry. The fields that constitute the SM Higgs doublet are among the pseudo-NambuGoldstone bosons (pNGBs) associated with the spontaneous breaking of the global $\mathrm{SU}(4) \times \mathrm{U}(1)$ symmetry down to $\mathrm{SU}(3) \times \mathrm{U}(1)$. Their mass is protected against one loop radiative corrections by the combination of the nonlinearly realized global symmetry and the discrete twin symmetry.

Since the original proposal, the MTH scenario has been further developed. Ultraviolet completions based on supersymmetry [15-20] and Higgs compositeness [21-23] that can raise the cutoff to the Planck scale have been proposed, and their collider implications studied [24,25]. Composite 
twin Higgs models have been shown to be consistent with precision electroweak constraints [26] and flavor bounds [27]. Various possibilities for the breaking of the discrete $Z_{2}$ symmetry and their effects on tuning have been studied [28-32]. More general global symmetry breaking patterns have also been investigated [33-36].

The cosmology of the MTH model is rather problematic. The twin sector is in thermal equilibrium with the SM in the early Universe up to temperatures of order a few $\mathrm{GeV}$ [7]. At lower temperatures the two sectors decouple, but the twin photon and twin neutrinos survive as thermal relics. These states contribute significantly to the total energy density in radiation, which conflicts with the bounds on dark radiation from the cosmic microwave background and big bang nucleosynthesis. This problem can be solved if the model is extended to realize a new contribution to the energy density of the SM sector after the two sectors have decoupled [37,38]. In general, this does not require additional breaking of the discrete $Z_{2}$ symmetry. Alternatively, the problem can be solved by introducing hard breaking of the $Z_{2}$ into the twin sector Yukawa couplings, thereby altering the spectrum of mirror states [39-42]. Once these cosmological bounds are satisfied puzzles like the nature of dark matter [39] or the baryon asymmetry [43] can be addressed.

Recently an alternative class of twin Higgs models, known as fraternal twin Higgs (FTH) models, has been proposed, in which the twin sector is more minimal than in the MTH, consisting of only those states that are required to address the hierarchy problem [44]. Specifically, the spectrum of light twin sector states includes only the third generation fermions, the electroweak gauge bosons, and the twin gluon. This framework naturally solves the cosmological problems of the MTH construction. It also leads to exotic collider signals since the lightest twin particles, the mirror glueballs, decay back to SM states, but with long lifetimes. Mirror glueballs can be produced in Higgs decays and will then decay far from the original interaction point, resulting in displaced vertices. The striking nature of these signals will allow the LHC to probe most of the preferred parameter space $[45,46]$. The proposed MATHUSLA detector $[47,48]$ is also expected to be sensitive to the displaced decays arising from this class of models. The FTH also contains several promising dark matter candidates [49-52] and has been put forward as a possible explanation of certain observed anomalies in large and small scale structure [53].

The only communication between the visible and twin sectors that is required by the twin Higgs framework is through the Higgs portal. After electroweak symmetry breaking the Higgs fields of the two sectors mix. The lighter mass eigenstate is identified with the $125 \mathrm{GeV}$ Higgs particle. As a consequence of the mixing it has suppressed couplings to SM fields, resulting in a production cross section that is smaller than the SM prediction.
This mixing also results in a contribution to the Higgs width from decays into invisible twin sector states. Unfortunately, while these signals are robust predictions of the MTH framework, they are not unique to it. They are expected to arise in any model in which the SM communicates with a light hidden sector through the Higgs portal.

If, however, the $Z_{2}$ symmetry is only softly broken, so that the Yukawa couplings in the two sectors are equal, the suppression in the Higgs production cross section and the Higgs invisible width are both determined by the mixing angle, leading to a prediction that can be tested by experiment [54]. This prediction does not apply to theories that exhibit hard breaking of $Z_{2}$, such as the FTH or MTH models in which the Yukawa couplings in the two sectors are different. The prediction can be understood as a consequence of the mirror nature of the model. Since it does not depend on the enhanced global symmetry of the Higgs sector, this prediction is not specific to the MTH construction, but applies more generally to any mirror model $[55,56]$ in which the discrete $Z_{2}$ symmetry is only softly broken, so that the Yukawa couplings in the two sectors are equal.

If the breaking of the global symmetry is realized linearly, the radial mode in the Higgs potential is present in the spectrum and constitutes a second portal between the twin and SM sectors. We refer to this state as the twin sector Higgs. As we now explain, a study of the properties of this particle at colliders, when combined with precision measurements of the light Higgs, can be used to overdetermine the form of the scalar potential, thereby confirming that it possesses an enhanced global symmetry as dictated by the twin Higgs mechanism.

In the case when the discrete $Z_{2}$ symmetry is only softly broken, the Higgs potential of the MTH model takes the form $^{1}$

$$
\begin{aligned}
V= & -\mu^{2}\left(H_{A}^{\dagger} H_{A}+H_{B}^{\dagger} H_{B}\right)+\lambda\left(H_{A}^{\dagger} H_{A}+H_{B}^{\dagger} H_{B}\right)^{2} \\
& +m^{2}\left(H_{A}^{\dagger} H_{A}-H_{B}^{\dagger} H_{B}\right)+\delta\left[\left(H_{A}^{\dagger} H_{A}\right)^{2}+\left(H_{B}^{\dagger} H_{B}\right)^{2}\right] .
\end{aligned}
$$

We distinguish the SM sector fields with the subscript $A$ and the twin sector fields with $B$. The terms in the top line of Eq. (1) respect both the global $\mathrm{SU}(4) \times \mathrm{U}(1)$ symmetry and the discrete $Z_{2}$ twin symmetry $A \leftrightarrow B$. The $m^{2}$ term explicitly breaks both the discrete and global symmetries, but only softly, and can naturally be smaller than $\mu^{2}$. The quartic term $\delta$ respects the $Z_{2}$ twin symmetry, but it violates the global symmetry. In order to realize the light Higgs as a pNGB and thereby obtain a significant reduction in finetuning relative to the SM the parameter $\delta$ that violates the global symmetry must be much smaller than $\lambda$, which is

\footnotetext{
${ }^{1}$ We employ the notation of [7].
} 
invariant under $\mathrm{SU}(4) \times \mathrm{U}(1)$. Similarly, $m^{2}$ must be much smaller than $\mu^{2}$.

The parameters in the Higgs potential must reproduce the mass of the light Higgs and the electroweak vacuum expectation value (VEV). This fixes two combinations of the four parameters. Two additional measurements are then required to fully determine the potential. At a lepton collider the production cross section and invisible width of the light Higgs can be determined to a precision of order one part in a hundred [57,58]. This covers the entire range of interest for the MTH and fixes a third combination of the parameters. Finally, the discovery of the twin sector Higgs particle at a given mass would pin down all four parameters in the Higgs potential. Once the potential has been specified, in the absence of further $Z_{2}$ violation, the production cross section, width and branching ratios of the twin sector Higgs are all robustly predicted. Therefore, a measurement of the rate to any SM final state overdetermines the system and constitutes a powerful consistency check on the form of the potential. These predictions remain true to a good approximation even in the presence of hard breaking of the $Z_{2}$ symmetry by the twin sector Yukawa couplings, provided that this breaking is not large enough to significantly alter the total width of the twin sector Higgs.

In the MTH framework, the breaking of the approximate global symmetry of the Higgs potential results in seven pNGBs. These include, in addition to the light Higgs, the longitudinal components of the $W^{ \pm}$and $Z$ bosons of both the SM and twin sector. It follows that in the limit that the global symmetry is exact, the couplings of the twin sector Higgs particle to all these seven states are the same, and they are set by the $\mathrm{SU}(4) \times \mathrm{U}(1)$ invariant quartic term in the Higgs potential. In particular, the couplings of this state to the SM Higgs, $W^{ \pm}$, and $Z$ are not suppressed by the mixing angle. In the limit that the twin sector Higgs particle is heavy, corresponding to the quartic term being large, its dominant decay modes are to these seven pNGBs. Furthermore, in the limit that the masses of the final state particles can be neglected, the branching ratio of the twin sector Higgs into each of these final states is the same. It follows that $W W, Z Z$ and di-Higgs are promising channels in which to search for the twin sector Higgs.

In this paper we study the prospects for the LHC and future colliders to discover the twin sector Higgs and determine the form of Higgs potential, thereby confirming the MTH framework. Discovery of the twin sector Higgs scalar at the LHC has been discussed previously $[18,35,44,59,60]$, but without the emphasis on determining the structure of the potential. We find that at the LHC, much of the range of parameter space in which the twin sector Higgs can be discovered is already disfavored by existing measurements of the couplings of the light Higgs. Only a restricted set of parameters leads to Higgs coupling deviations and a twin sector Higgs signal that can both be measured at the LHC. We find that the high energy stages of linear colliders such as the International Linear Collider (ILC) [61] or Compact Linear Collider (CLIC) [62] are expected to have much greater reach for the twin sector Higgs. These colliders are also projected to measure the invisible width of the light Higgs to percent level precision [57,58]. Combining the measurements of the twin sector Higgs with precision studies of the couplings of the light Higgs results in much greater ability to confirm the MTH construction.

The outline of this paper is as follows. In Sec. II we describe the scalar sector of the MTH in detail and develop the notation we use in the rest of the paper. In Sec. III, we determine the reach of the LHC for the twin sector Higgs. In Sec. IV, we study the potential for the ILC and CLIC to discover the twin sector Higgs, and we determine its couplings. We conclude in Sec. V.

\section{THE SCALAR SECTOR OF THE MIRROR TWIN HIGGS}

This section outlines the dynamics of the scalar sector in the MTH framework. We begin by analyzing the Higgs potential shown in Eq. (1). It is convenient to employ an exponential parametrization of the scalar degrees of freedom. Accordingly, we define an object $H$ which transforms linearly under $\mathrm{SU}(4) \times \mathrm{U}(1)$,

$$
H=\left(\begin{array}{c}
H_{A} \\
H_{B}
\end{array}\right)=\exp \left(\frac{i}{f} \Pi\right)\left(\begin{array}{c}
0 \\
0 \\
0 \\
f+\frac{\sigma}{\sqrt{2}}
\end{array}\right) .
$$

Here $f$ is the symmetry breaking VEV, and $\Pi$ is given, in unitary gauge where all the $B$ sector pNGBs have been eaten by the corresponding vector bosons, by

$$
\Pi=\left(\begin{array}{ccc|c}
0 & 0 & 0 & i h_{1} \\
0 & 0 & 0 & i h_{2} \\
0 & 0 & 0 & 0 \\
\hline-i h_{1}^{*} & -i h_{2}^{*} & 0 & 0
\end{array}\right) .
$$

Expanding the exponential we obtain

$$
H=\left(\begin{array}{c}
\frac{\boldsymbol{h}}{\sqrt{\boldsymbol{h}^{\dagger} \boldsymbol{h}}}\left(f+\frac{\sigma}{\sqrt{2}}\right) \sin \left(\frac{\sqrt{\boldsymbol{h}^{\dagger} \boldsymbol{h}}}{f}\right) \\
0 \\
\left(f+\frac{\sigma}{\sqrt{2}}\right) \cos \left(\frac{\sqrt{\boldsymbol{h}^{\dagger} \boldsymbol{h}}}{f}\right)
\end{array}\right),
$$

where $\boldsymbol{h}=\left(h_{1}, h_{2}\right)^{\mathrm{T}}$ is the SM Higgs doublet. Proceeding to unitary gauge in the SM sector with $h_{1}=0$ and $h_{2}=$ $(v+h) / \sqrt{2}$ leads to 


$$
\begin{aligned}
& H_{A}=\left(\begin{array}{c}
0 \\
\left(f+\frac{\sigma}{\sqrt{2}}\right) \sin \left(\frac{v+h}{\sqrt{2} f}\right)
\end{array}\right), \\
& H_{B}=\left(\begin{array}{c}
0 \\
\left(f+\frac{\sigma}{\sqrt{2}}\right) \cos \left(\frac{v+h}{\sqrt{2} f}\right)
\end{array}\right),
\end{aligned}
$$

and allows us to write the potential as

$$
\begin{aligned}
V= & f^{2}\left(1+\frac{\sigma}{\sqrt{2} f}\right)^{2}\left[-\mu^{2}-m^{2} \cos \left(\frac{\sqrt{2}(v+h)}{f}\right)\right] \\
& +f^{4}\left(1+\frac{\sigma}{\sqrt{2} f}\right)^{4}\left[\lambda+\delta-\frac{\delta}{2} \sin ^{2}\left(\frac{\sqrt{2}(v+h)}{f}\right)\right] .
\end{aligned}
$$

It is convenient to define the angular variable $\vartheta \equiv v /(\sqrt{2} f)$. In terms of $\vartheta$ and $f$, the VEVs in the visible and twin sectors are given by

$$
v_{\mathrm{EW}} \equiv \sqrt{2} f \sin \vartheta, \quad v_{B} \equiv \sqrt{2} f \cos \vartheta
$$

The equations of motion for $\sigma$ and $h$ take the form

$$
\begin{aligned}
\mu^{2}+m^{2} \cos (2 \vartheta) & =f^{2}\left[2(\lambda+\delta)-\delta \sin ^{2}(2 \vartheta)\right], \\
m^{2} & =\delta f^{2} \cos (2 \vartheta)
\end{aligned}
$$

We see from the second equation that in the $Z_{2}$ symmetric limit $\left(m^{2}=0\right)$ the mixing is maximal, with $v_{\mathrm{EW}}=v_{B}$ and $\theta=\pi / 4$. This would mean that the observed $125 \mathrm{GeV}$ Higgs boson couples just as strongly to the twin sector as to the SM, which conflicts with current data. Therefore we need $m^{2}>0$, which corresponds to $\vartheta<\pi / 4$, to obtain realistic phenomenology. We have assumed here that $\delta>0$, which is required for stable vacuum [7]. Combining the equations of motion we obtain

$$
\mu^{2}=f^{2}(2 \lambda+\delta)
$$

The mass eigenstates $h_{-}$and $h_{+}$are linear combinations of $h$ and $\sigma$,

$$
\left(\begin{array}{l}
h_{-} \\
h_{+}
\end{array}\right)=\left(\begin{array}{cc}
\cos \theta & \sin \theta \\
-\sin \theta & \cos \theta
\end{array}\right)\left(\begin{array}{l}
h \\
\sigma
\end{array}\right) \text {. }
$$

The mixing angle $\theta$ is given by

$\sin (2 \theta)=\frac{2 f^{2} \delta \sin (4 \vartheta)}{m_{+}^{2}-m_{-}^{2}}, \quad \cos (2 \theta)=\frac{4 f^{2}\left[\lambda+\delta \cos ^{2}(2 \vartheta)\right]}{m_{+}^{2}-m_{-}^{2}}$

The mass eigenvalues $m_{+}$and $m_{-}$are given by

$$
m_{ \pm}^{2}=2 f^{2}\left[\lambda+\delta \pm \sqrt{\lambda^{2}+\delta(2 \lambda+\delta) \cos ^{2}(2 \vartheta)}\right]
$$

We can express $\lambda^{2}$ in terms of $m_{+}, m_{-}$, and $\vartheta$,

$$
\lambda^{2}=\frac{1}{16 f^{4}}\left[\left(m_{+}^{2}-m_{-}^{2}\right)^{2}-4 \cot ^{2}(2 \vartheta) m_{+}^{2} m_{-}^{2}\right] .
$$

Note that in order to keep $\lambda^{2} \geq 0$ we must have

$$
\frac{m_{+}}{m_{-}} \geq|\cot (2 \vartheta)|+|\csc (2 \vartheta)| \text {. }
$$

Since $\vartheta<\pi / 4$ we can drop the absolute value symbols to obtain

$$
\frac{m_{+}}{m_{-}} \geq \cot \vartheta=\frac{v_{B}}{v_{\mathrm{EW}}}=\frac{m_{T}}{m_{t}} .
$$

Here $m_{t}$ represents the mass of the top quark and $m_{T}$ the mass of the its twin counterpart, the top partner. This inequality places a lower bound on the mass of the twin sector Higgs relative to the mass of the top partner.

\section{A. Higgs couplings in the twin Higgs framework}

The couplings of the Higgs fields to the $W$ and $Z$ gauge bosons arise from the kinetic terms,

$$
\left|\left(\partial_{\mu}+i g W_{\mu, A}^{(j)}+\frac{i}{2} g^{\prime} B_{\mu, A}\right) H_{A}\right|^{2}+(A \rightarrow B) .
$$

Here the $W_{\mu}^{(1,2,3)}$ are the three gauge bosons of $\mathrm{SU}(2)_{L}$ and $B_{\mu}$ that of hypercharge. The resulting $W$ boson masses are given by

$$
\begin{aligned}
& M_{W_{A}}^{2}=\frac{f^{2} g^{2}}{2} \sin ^{2} \vartheta=\frac{g^{2} v_{\mathrm{EW}}^{2}}{4}, \\
& M_{W_{B}}^{2}=\frac{f^{2} g^{2}}{2} \cos ^{2} \vartheta=\frac{g^{2} v_{B}^{2}}{4} .
\end{aligned}
$$

The $Z$ boson masses are related to these by the usual factor of $\cos \theta_{W}$. In unitary gauge, the couplings of the light Higgs to the $W$ and $Z$ bosons that result from Eq. (18) take the form (see the Appendix for details)

$$
\begin{aligned}
& 2 \frac{h_{-}}{v_{\mathrm{EW}}} \cos (\vartheta-\theta)\left[M_{W_{A}}^{2} W_{A \mu}^{+} W_{A}^{\mu-}+\frac{1}{2} M_{Z_{A}}^{2} Z_{A \mu} Z_{A}^{\mu}\right] \\
& \quad-2 \frac{h_{-}}{v_{B}} \sin (\vartheta-\theta)\left[M_{W_{B}}^{2} W_{B \mu}^{+} W_{B}^{\mu-}+\frac{1}{2} M_{Z_{B}}^{2} Z_{B \mu} Z_{B}^{\mu}\right] .
\end{aligned}
$$

The corresponding expression for the heavy Higgs is given by 


$$
\begin{aligned}
& 2 \frac{h_{+}}{v_{\mathrm{EW}}} \sin (\vartheta-\theta)\left[M_{W_{A}}^{2} W_{A \mu}^{+} W_{A}^{\mu-}+\frac{1}{2} M_{Z_{A}}^{2} Z_{A \mu} Z_{A}^{\mu}\right] \\
& \quad+2 \frac{h_{+}}{v_{B}} \cos (\vartheta-\theta)\left[M_{W_{B}}^{2} W_{B \mu}^{+} W_{B}^{\mu-}+\frac{1}{2} M_{Z_{B}}^{2} Z_{B \mu} Z_{B}^{\mu}\right] .
\end{aligned}
$$

The couplings of the SM and twin sector fermions to the Higgs fields emerge from the Yukawa interactions,

$$
\begin{aligned}
& -Y_{u}^{i j} \bar{Q}_{A \alpha}^{i} \epsilon^{\alpha \beta} H_{A \alpha}^{\dagger} u_{A}^{j}-Y_{d}^{i j} \bar{Q}_{A \alpha}^{i} H_{A}^{\alpha} d_{A}^{j}-Y_{\ell}^{i j} \bar{L}_{A \alpha}^{i} H_{A}^{\alpha} e_{A}^{j} \\
& \quad+(A \rightarrow B) .
\end{aligned}
$$

Here $i$ and $j$ represent flavor indices, while $\alpha$ and $\beta$ are $\mathrm{SU}(2)$ indices. This results in the fermions acquiring masses,

$$
m_{f_{A}}=Y_{f} v_{\mathrm{EW}} / \sqrt{2}, \quad m_{f_{B}}=Y_{f} v_{B} / \sqrt{2} .
$$

The couplings of the light Higgs to the fermions $f$ that result from Eq. (22) take the form (for details see the Appendix)

$$
-h_{-}\left[\bar{f}_{A} f_{A} \frac{m_{f_{A}}}{v_{\mathrm{EW}}} \cos (\vartheta-\theta)-\bar{f}_{B} f_{B} \frac{m_{f_{B}}}{v_{B}} \sin (\vartheta-\theta)\right] .
$$

The corresponding couplings of the twin sector Higgs are given by

$$
-h_{+}\left[\bar{f}_{A} f_{A} \frac{m_{f_{A}}}{v_{\mathrm{EW}}} \sin (\vartheta-\theta)+\bar{f}_{B} f_{B} \frac{m_{f_{B}}}{v_{B}} \cos (\vartheta-\theta)\right] .
$$

We see from this that, in general, the masses of the visible and twin sector particles are related by

$$
m_{B}^{2}=m_{A}^{2} \frac{v_{B}^{2}}{v_{\mathrm{EW}}^{2}}=m_{A}^{2} \cot ^{2} \vartheta .
$$

The couplings of the light Higgs $h_{-}$to visible sector particles are related to the corresponding couplings in the SM by

$$
g_{h_{-} \mathrm{SM}}=g_{\mathrm{SM}} \cos (\vartheta-\theta),
$$

while the corresponding couplings of the twin sector Higgs $h_{+}$are given by

$$
g_{h_{+} \mathrm{SM}}=g_{\mathrm{SM}} \sin (\vartheta-\theta) .
$$

The couplings of the Higgs fields to the twin sector are also related to the corresponding SM couplings. The light Higgs couples to twin states as it does to SM states, but with the replacement $v_{\mathrm{EW}} \rightarrow v_{B}$, and a factor of $\sin (\vartheta-\theta)$. The couplings of $h_{+}$to twin states are again those of the
$\mathrm{SM}$, but with the replacement $v_{\mathrm{EW}} \rightarrow v_{B}$, and a factor of $\cos (\vartheta-\theta)$.

As detailed in the Appendix, loop induced couplings to pairs of photons or gluons result from a single tree level coupling between a Higgs and the fermion or vector in the loop, leading to the same $\cos (\vartheta-\theta)$ or $\sin (\vartheta-\theta)$ modification. However, the decays of the heavy Higgs are also a function of the mass of the Higgs. So, the decay widths of the twin sector Higgs to visible sector states are those of a SM Higgs with mass $m_{+}$multiplied by $\sin ^{2}(\vartheta-\theta)$.

\section{B. Decays of the twin sector Higgs}

With the couplings of the twin sector Higgs in hand, we are now in a position to compute its branching ratios. From Fig. 1 we see that for $m_{+}<m_{T}$ the twin sector Higgs decays primarily to SM electroweak gauge bosons. Once $m_{+} \gtrsim m_{T}$ decays to the $W_{B}$ and $Z_{B}$ bosons become kinematically accessible, and begin to play an important role. For heavy Higgs masses we see that visible decays into $W W, Z Z$ and di-Higgs dominate, together with invisible decays. Their respective contributions to the branching ratio are roughly $2 / 7,1 / 7,1 / 7,3 / 7$, exactly as expected from symmetry arguments. For small $m_{+}$we approach the edge of potential stability. Near the edge $\vartheta \sim \theta$, leading to sequestering of the two sectors. However, this tuned region does not correspond to a twin Higgs-like potential since it is not approximately $\mathrm{SU}(4)$ symmetric, as we show in Sec. II C.

Clearly, the potential in Eq. (1) is defined by four parameters. The measured values of $v_{\mathrm{EW}}$ and $m_{h}=m_{-}$ already constrain this system. Measuring deviations in the Higgs couplings to SM fields determines $\cos (\vartheta-\theta)$. Currently, the LHC has measured some Higgs couplings to $\sim 10 \%$ accuracy [63], and it is expected to reach $\sim 5 \%$ precision by the end of the high luminosity run [64]. Linear electron positron colliders can reduce the uncertainty to better than $1 \%$. Measuring the mass of the twin

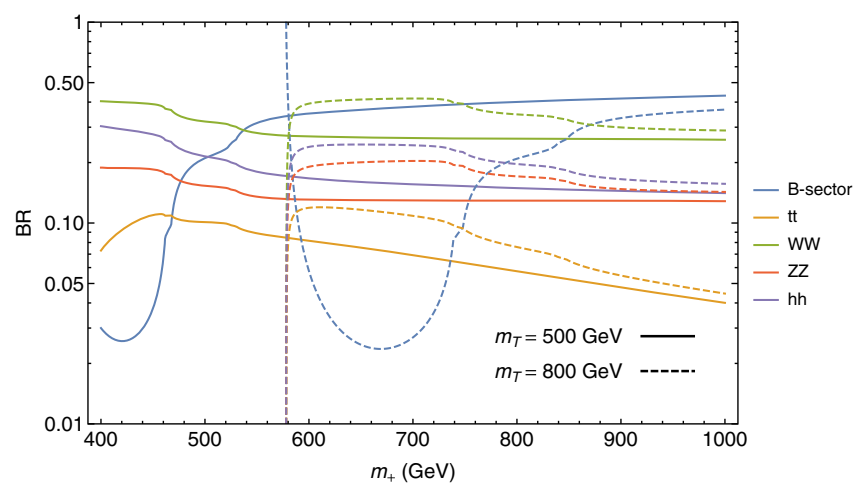

FIG. 1. Branching ratios of the heavy twin sector Higgs scalar to various SM final states and the twin sector. 
sector Higgs $m_{+}$would then completely determine the potential. The width and branching ratios of the heavy Higgs are then completely specified. Therefore, with the mass in hand, measuring the twin sector Higgs rate into one or more visible states constitutes a powerful test of the twin Higgs framework.

Our discussion till now has focused on the case in which the discrete $Z_{2}$ symmetry is only softly broken. However, a small hard breaking of the discrete symmetry by the twin sector Yukawa couplings allows a simple resolution of the cosmological problems associated with the MTH framework. We therefore briefly consider the implications of a hard breaking of the discrete symmetry in the Yukawa sector.

Once the twin sector Yukawa couplings are allowed to vary, the invisible decay widths of both the light Higgs and the heavy Higgs are affected. In the case of the light Higgs, by measuring the total rate into both visible and invisible final states, it is still possible to extract $\cos (\vartheta-\theta)$. In the case of the twin sector Higgs, however, without any knowledge of the branching ratio into the twin sector, it is no longer possible to predict the rate into visible states. However, for large twin sector Higgs masses, unless the hard breaking of the discrete symmetry by the Yukawas is very large, the primary decay modes are expected to be the same as in the soft breaking case. This can be seen in Fig. 2, where the invisible branching ratio of the twin sector Higgs in the FTH model has been plotted against $m_{+}$for two different values of the twin bottom Yukawa coupling and compared against the invisible branching ratio in the MTH model. We see that once $m_{+}>m_{T}$, the different curves quickly converge towards $3 / 7$, the theoretical prediction. We conclude that for heavy twin sector Higgs bosons, if the hard breaking of the discrete symmetry is small, the

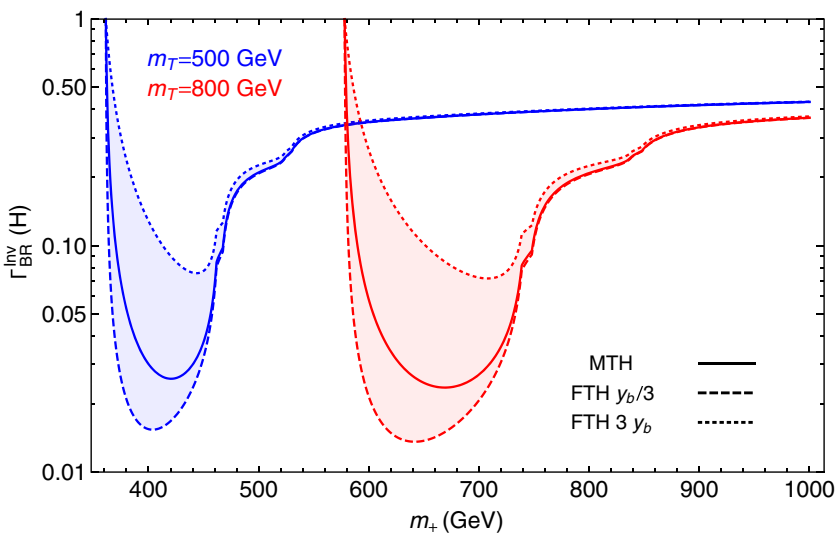

FIG. 2. Branching fraction of the twin sector Higgs to twin states as a function of its mass for the MTH (solid curves) and FTH (dashed and dotted curves) models. The blue (red) curves correspond to a mirror top mass of 500 (1000) GeV. In the FTH model the twin bottom Yukawa varies from one third the SM value (dashed line) to three times the SM value (dotted line). predictions of the MTH continue to hold to a good approximation.

\section{Fine-tuning in the model}

In the twin Higgs framework, the global symmetry of the Higgs sector is ultimately what protects the mass of the light Higgs from large radiative corrections. Therefore, the SU(4) violating parameters $m^{2}$ and $\delta$ in the Higgs potential, Eq. (1), must be small compared to their SU(4) invariant counterparts, $\mu^{2}$ and $\lambda$, in order for the visible sector Higgs to be naturally light. Dividing Eq. (10) by Eq. (11) we obtain

$$
\frac{m^{2}}{\mu^{2}}=\frac{\frac{\delta}{\lambda} \cos 2 \vartheta}{2+\frac{\delta}{\lambda}},
$$

which shows that as long as $\delta \ll \lambda$ the potential is approximately SU(4) symmetric, and corresponds to a twin Higgs potential.

To determine the fine-tuning in this model, note that the parameter $\mu^{2}$ receives radiative corrections from the top loop, just as the Higgs mass parameter in the SM does. Assuming a cutoff $\Lambda$, the fine-tuning associated with the sensitivity of $\mu^{2}$ to the cutoff is given by

$$
\frac{3 Y_{t}^{2}}{8 \pi^{2}} \frac{\Lambda^{2}}{\mu^{2}}
$$

If the potential is approximately $\mathrm{SU}(4)$ invariant, so that $\lambda \gg \delta$, we can combine Eqs. (11) and (14) to obtain $2 \mu^{2} \simeq m_{+}^{2}$. Then the expression for this fine-tuning reduces to

$$
\frac{3 Y_{t}^{2}}{4 \pi^{2}} \frac{\Lambda^{2}}{m_{+}^{2}} .
$$

Now, it follows from Eqs. (29) and (11) that the electroweak scale $v_{\mathrm{EW}}$ is related to $\mu^{2}$ as

$$
v_{\mathrm{EW}}^{2}=\frac{\mu^{2}}{2 \lambda+\delta}-\frac{m^{2}}{\delta} .
$$

Then the sensitivity of the electroweak scale to the parameter $\mu^{2}$ is given by

$$
\frac{\mu^{2}}{v_{\mathrm{EW}}^{2}} \frac{\partial v_{\mathrm{EW}}^{2}}{\partial \mu^{2}}=\frac{\mu^{2}}{v_{\mathrm{EW}}^{2}(2 \lambda+\delta)}=\frac{f^{2}}{v_{\mathrm{EW}}^{2}} \simeq \frac{m_{T}^{2}}{2 m_{t}^{2}} .
$$

The sensitivity of the electroweak scale to the cutoff in this model is obtained from Eqs. (31) and (33) as

$$
\frac{3 Y_{t}^{2}}{4 \pi^{2}} \frac{\Lambda^{2}}{m_{+}^{2}} \frac{m_{T}^{2}}{2 m_{t}^{2}} .
$$


In comparison, the sensitivity of the electroweak scale to the cutoff in the SM is given by

$$
\frac{3 Y_{t}^{2}}{4 \pi^{2}} \frac{\Lambda^{2}}{m_{-}^{2}}
$$

where $m_{-}$is $125 \mathrm{GeV}$, the mass of the light Higgs. Then the improvement in fine-tuning with respect to the SM for the same cutoff is obtained by taking the ratio of Eq. (35) to Eq. (34),

$$
2 \frac{m_{+}^{2}}{m_{-}^{2}} \frac{m_{t}^{2}}{m_{T}^{2}}
$$

We see from this that, for a given top partner mass, a heavier twin sector Higgs is preferred. We require $m_{+}>$ $m_{T}$ to obtain any significant improvement in fine-tuning with respect to the SM.

\section{CURRENT AND FUTURE LHC REACH}

As in all pNGB Higgs models, the twin Higgs framework predicts reduced couplings of the light Higgs to SM states, resulting in a suppression of the Higgs production cross section. This, together with Higgs decays into invisible twin sector final states, results in fewer Higgs events. The resulting LHC constraints on the MTH model were calculated in [54], but this analysis assumed that the

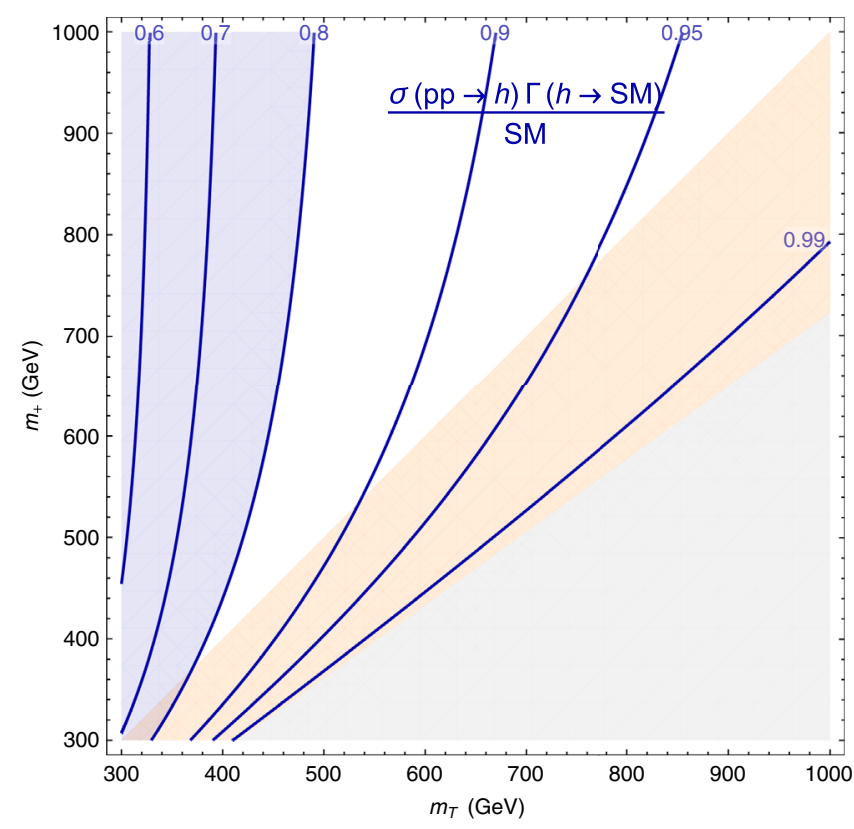

FIG. 3. The region with stable vacuum but with $m_{+}<m_{T}$, and hence the tuning is not significantly improved over the SM, is shaded orange. The grey region does not allow a stable vacuum. The blue shaded region is disfavored by LHC Higgs coupling measurements. radial mode was heavy, with mass near the cutoff of the model. Here we determine the suppression of Higgs rates relative to the SM, taking into account the finite mass of the radial mode. In Fig. 3 we display contours of the ratio of Higgs event rates to visible sector states in the MTH model relative to Higgs event rates in the SM. We see that for lighter radial modes the deviation from the SM value decreases.

The LHC has already measured the one sigma Higgs couplings to EW gauge bosons to 10\% [63]. This implies that the region to the left of the 0.8 contour is already ruled out. The HL-LHC is expected to probe up to the 0.9 contour. As the figure shows, the LHC Higgs coupling measurements cannot fully probe the parameter space of the MTH model.

The LHC has a strong experimental program directly searching for heavy Higgs-like scalars $H$, but present search limits are weaker than Higgs coupling constraints. However, both ATLAS [65] and CMS [66] have estimated the high luminosity reach for a heavy scalar in the $H \rightarrow Z Z \rightarrow 4 \ell$ channel. In Fig. 4 we see the exclusion curves for both detectors as well as the discovery region for CMS. The reach for ATLAS is expected to be comparable. We use the ATLAS

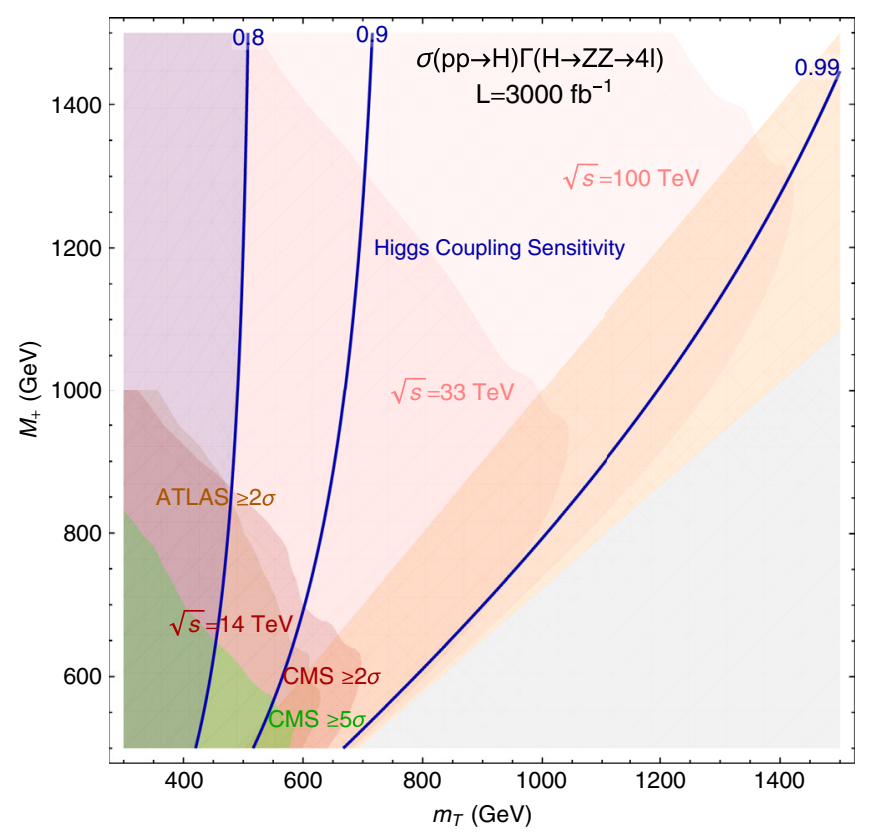

FIG. 4. Exclusion bound on the twin Higgs model in the mass plane of twin top and twin sector Higgs for the high luminosity LHC run, as well as for future hadron colliders with center of mass energies of 33 and $100 \mathrm{TeV}$. The extrapolation for the HLLHC is made by ATLAS and CMS for the $H \rightarrow Z Z \rightarrow 4 \ell$ process. Blue contours denote variation in Higgs couplings, with the region to the left of 0.8 already excluded by LHC measurements. The orange region does not significantly improve tuning compared to the SM. The extrapolation to future hadron colliders is estimated using Fig. 3 of Ref. [59]. See text for further details. 
exclusion numbers as well as the CMS exclusion and $5 \sigma$ numbers from Fig. 3 of [67]. We translate the bounds into our framework for any chosen masses of the twin sector Higgs and twin sector top by first rescaling the SM Higgs cross section provided by the LHC Higgs Cross Section Working Group [68,69] by the factor of $\sin ^{2}(\vartheta-\theta)$. Then using the results collected in the Appendix we include the branching fraction of $h_{+}$into ZZ. This leads to the red and green shaded contours in Fig. 4. These searches can probe twin sector Higgs masses as heavy as a $\mathrm{TeV}$, but only if the twin top is light. Unfortunately, the greater part of this region is already excluded by Higgs coupling measurements. It is only in a limited region of allowed parameter space that the LHC will be able to discover the twin sector Higgs.

If Higgs coupling deviations are observed at the HL-LHC and a heavy scalar is found in the four lepton search, the entire Higgs potential will be specified. This leads to a specific prediction for the $H \rightarrow Z Z$ rate, which can be compared with the experimental results, explicitly testing the mechanism. Unfortunately, the region of parameter space in which such a scenario can play out is rather limited.

For completeness we also include in Fig. 4 an estimate for the sensitivity at future hadron colliders, with center of mass energies of 33 and $100 \mathrm{TeV}$. In order to estimate the signal cross section in our model at future colliders, we scale up the cross section for the twin sector Higgs at $14 \mathrm{TeV}$ (obtained as described above) by the appropriate ratio of PDF luminosities, using Fig. 3 of Ref. [70] for $33 \mathrm{TeV}$ and Table 2 of Ref. [71] for $100 \mathrm{TeV}$. We then compare the cross section (times branching ratio) thus obtained to Fig. 3 of Ref. [59], where the sensitivity at these colliders to heavy scalars decaying to $Z Z$ was estimated, under the assumption that the background is primarily $q-\bar{q}$ initiated. As can be seen from Fig. 4, going to higher energy significantly extends the region of parameter space where hadron colliders have sensitivity to the twin sector Higgs.

\section{LINEAR COLLIDER REACH}

In this section, we discuss the potential for the next generation of linear colliders to discover the twin sector
Higgs, and determine the parameters in the scalar sector of the MTH model. For concreteness, we focus on the ILC and CLIC proposals. These colliders possess two advantages with respect to the LHC.

(i) Both of these machines will be able to measure the couplings of the light Higgs to better than 1\%, which, as can be seen from Fig. 3, covers most of the preferred parameter space.

(ii) Because of their much lower backgrounds, these colliders potentially have much greater reach for the twin sector Higgs.

As explained earlier, the mass of the twin sector Higgs, together with measurements of the deviations in couplings of the light Higgs, completely specifies the scalar potential of the MTH model. Then, a measurement of the rate of twin sector Higgs events into any SM final state overdetermines the scalar potential and constitutes a powerful test of this framework.

For our analysis, we focus on benchmark scenarios motivated by the ILC and CLIC proposals. For the high energy ILC, which is a $1 \mathrm{TeV}$ machine, we consider two benchmark scenarios corresponding to $1 \mathrm{ab}^{-1}$ and $3 \mathrm{ab}^{-1}$ of integrated luminosity. The CLIC benchmark corresponds to a $1.5 \mathrm{TeV}$ machine with an integrated luminosity of $1.5 \mathrm{ab}^{-1}$. Signals are generated in MADGRAPH5 [72] and showered with Pythia8 [73]. We use the DelPhES3 [74] detector simulator with the anti- $k_{\mathrm{T}}$ clustering algorithm [75] and FASTJET [76] library to simulate the detector. We use the DELPHES card based on the ILC construction outlined in [77]. A simulation of the CLIC detector is not yet available, but it is expected to be qualitatively similar.

The branching ratios shown in Fig. 1 show that of the twin sector Higgs's visible decay products, $W W$ is the largest. However, the $W W$ background is prohibitively large. Instead we focus on decays to di-Higgs, for which the background is orders of magnitude smaller, making the process $h_{+} \rightarrow h_{-} h_{-} \rightarrow 4 b$ very attractive. While the $4 b$ final state is difficult to extract from background at a hadron collider, the comparatively clean environment of a lepton machine is admirably suited to such a search.

Figure 5 displays the dominant Higgs production processes at lepton colliders. Our analysis employs the dominant Higgs production process at high energies, which is $W W$ fusion. Our study required at least three jets, each required to have $p_{T}>20 \mathrm{GeV}$ and $|\eta|<2.5$.

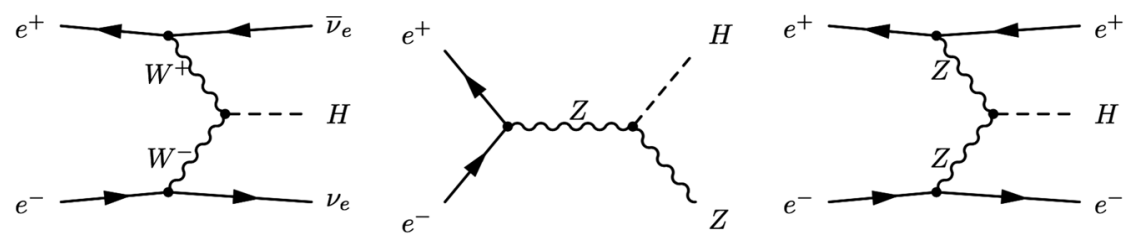

FIG. 5. Dominant Higgs production mechanisms at lepton colliders. 

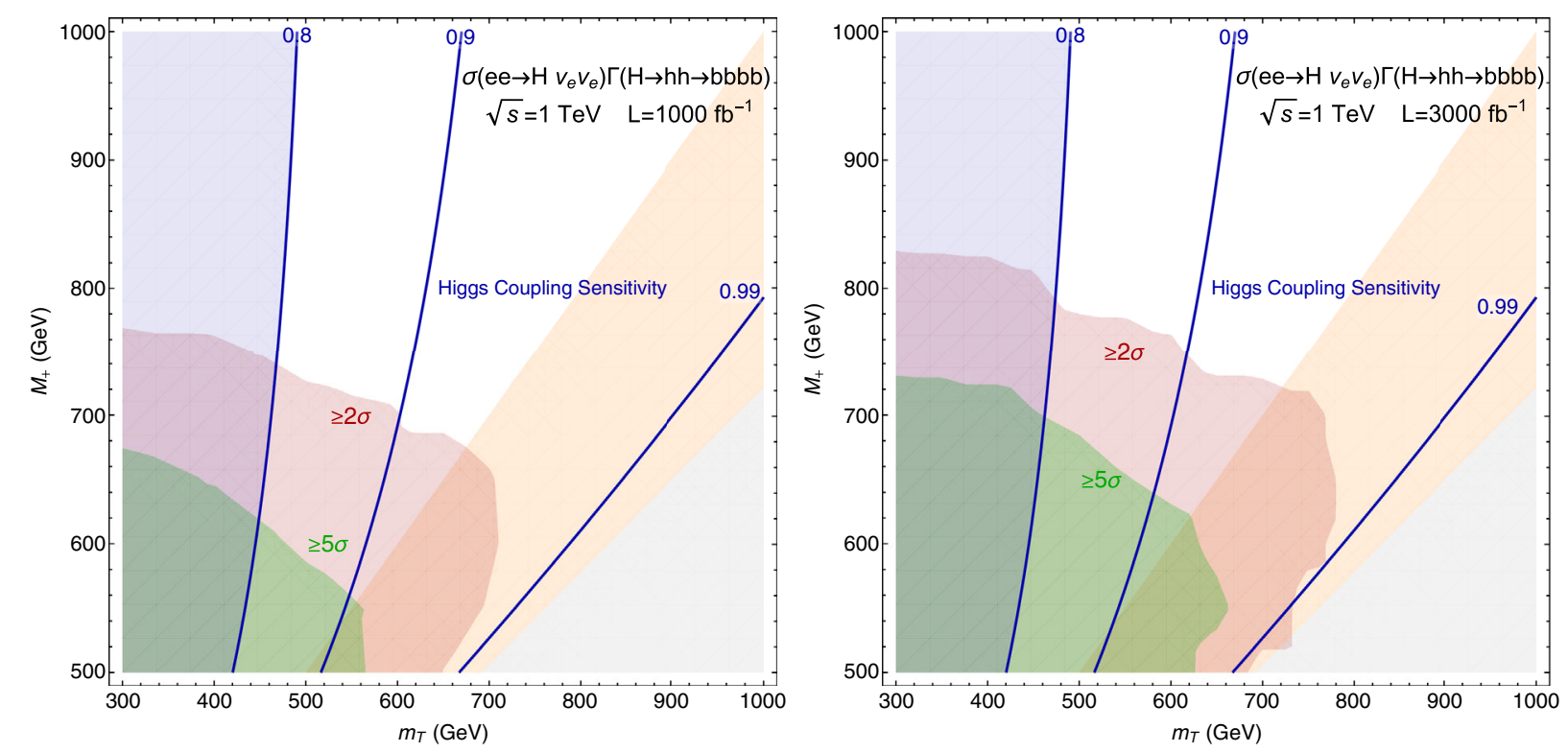

FIG. 6. Results for both the ILC $1 \mathrm{ab}^{-1}$ (left) and $3 \mathrm{ab}^{-1}$ (right) benchmark linear collider scenarios for $W$ fusion to heavy twin sector Higgs decaying to di-Higgs to four $b$ 's. As in Fig. 4, the blue contours indicate deviation in Higgs couplings, with the region to the left of 0.8 excluded by current measurements. The gray region does not provide a stable vacuum.

In addition, we demand three $b$-tags and that the jets reconstruct two on-shell Higgs bosons, with on-shell window $(75 \mathrm{GeV}, 135 \mathrm{GeV})$. We considered invariant mass bins that contained at least $85 \%$ of the signal events in the four bins surrounding the peak that passed the previous cuts. These bins were taken to be several tens of $\mathrm{GeV}$ wide to accommodate the expected jet energy resolution. In Fig. 6 we see the results for both the ILC benchmarks. Comparing this figure to the LHC results in Fig. 4, we find that the reach of the ILC with $1 \mathrm{ab}^{-1}$ is comparable to that of the HL-LHC. With $3 \mathrm{ab}^{-1}$ the ILC will be able to discover the twin sector Higgs for a greater range of twin top and twin sector Higgs masses than the LHC. The higher energy and increased luminosity of the CLIC benchmark allow even greater opportunity to discover the twin sector Higgs, as can be seen from Fig. 7.

Finally, we quantify the confidence with which the twin Higgs mechanism can be confirmed as follows. For a given parameter point, we calculate the uncertainty in the number of observed events after the cuts described above (due to Poisson statistics), and we also estimate the uncertainty in the expected number of events at that parameter point, where the leading contribution is the uncertainty in the value of $\sin ^{2}(\vartheta-\theta)$ arising from Higgs coupling measurements. In particular, we assume that $\kappa_{Z}$, the multiplicative factor that measures the deviation of the Higgs coupling to the Z-boson, can be measured with a precision of $0.5 \%$ [64]. Combining the uncertainties in the number of expected and observed events, we arrive at the fractional uncertainty in the ratio of observed to expected events, centered around the value 1 . The fractional uncertainty is plotted for the $\operatorname{ILC}\left(\int d t \mathcal{L}=3 \mathrm{ab}^{-1}\right)$ and CLIC $\left(\int d t \mathcal{L}=1.5 \mathrm{ab}^{-1}\right)$ benchmarks in Fig. 8.

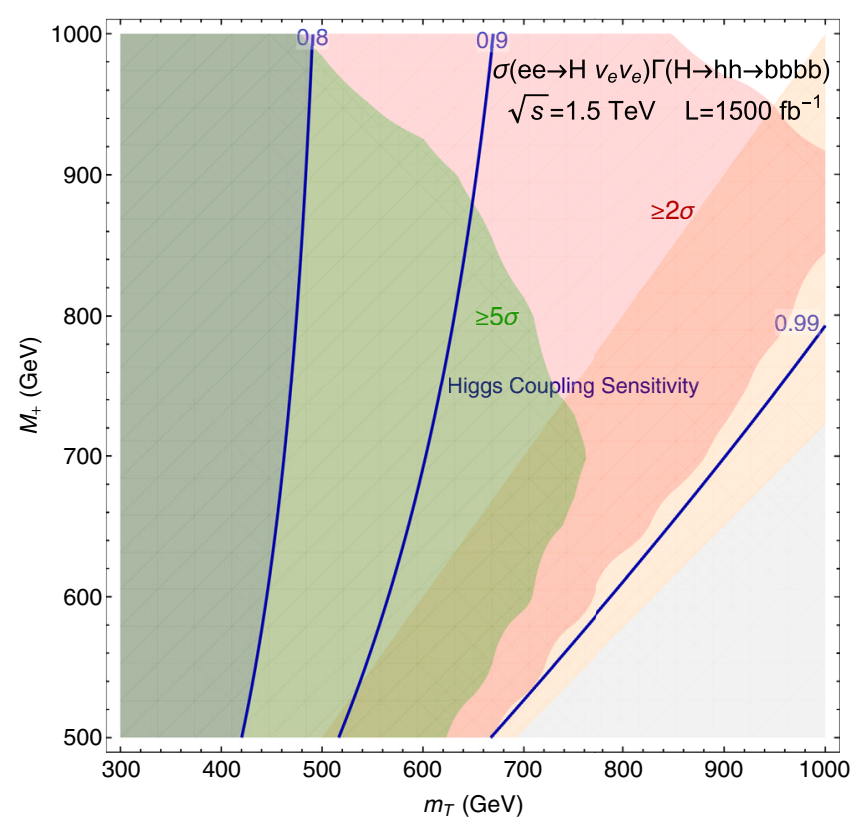

FIG. 7. Results for both the CLIC benchmark linear collider scenario for $W$ fusion to heavy twin sector Higgs decaying to diHiggs to four $b$ 's. As in Fig. 4, the blue contours indicate deviation in Higgs couplings, with the region to the left of 0.8 excluded by current measurements. The gray region does not provide a stable vacuum. 

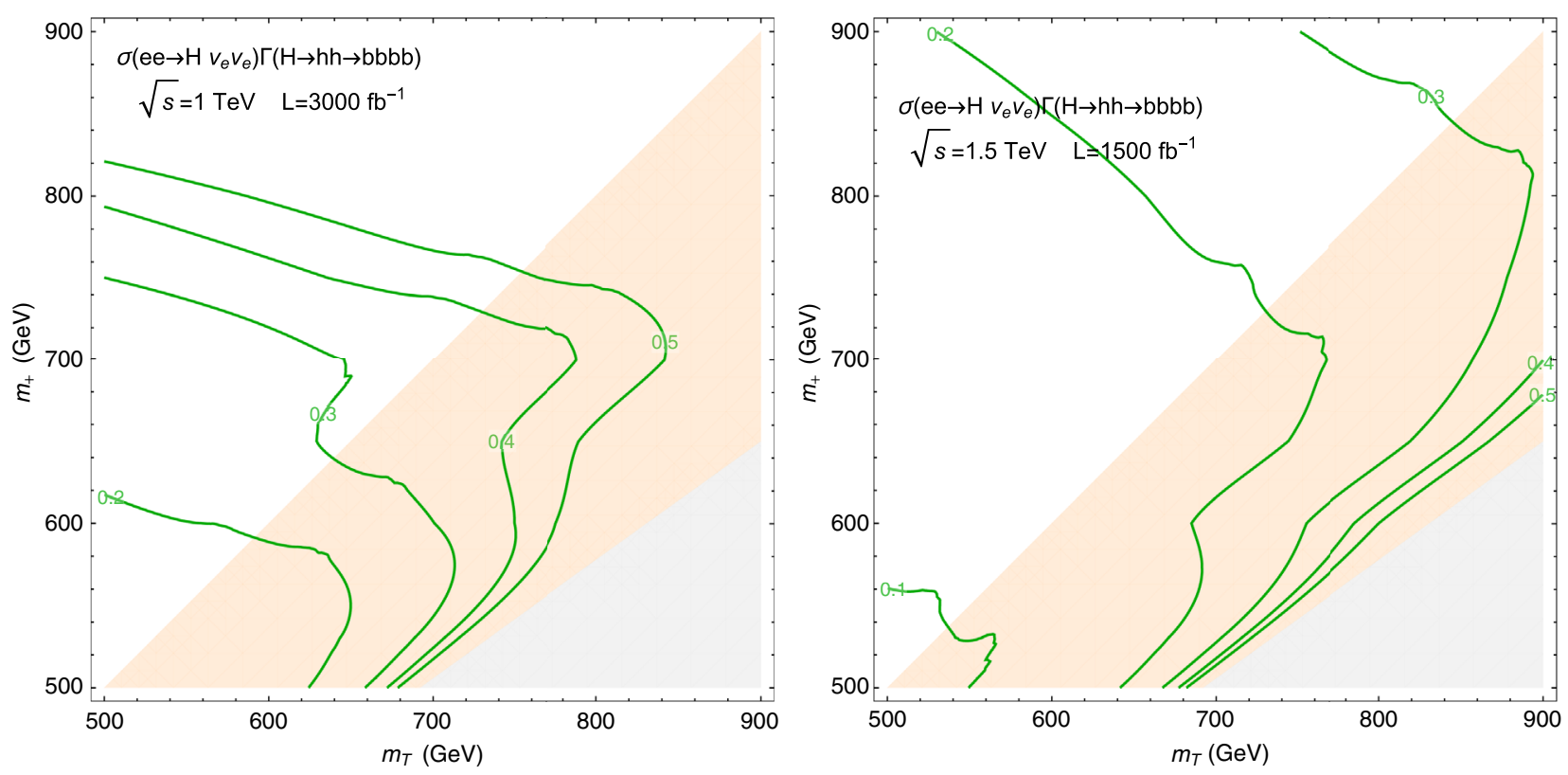

FIG. 8. The uncertainty in the ratio of observed and expected events, centered around the value 1 , for the ILC $\left(\int d t \mathcal{L}=3 \mathrm{ab}^{-1}\right)($ left) and CLIC $\left(\int d t \mathcal{L}=1.5 \mathrm{ab}^{-1}\right)$ (right) benchmarks as a function of $m_{+}$and $m_{T}$. See main text for additional details.

\section{CONCLUSION}

The twin Higgs mechanism protects the mass of the Higgs against radiative corrections without requiring new particles charged under the SM gauge groups. In this framework, the light Higgs emerges as the pNGB associated with the breaking of a global symmetry, and its mass is protected against quantum effects by a combination of the global symmetry and a discrete $Z_{2}$ symmetry. If the breaking of the global symmetry is realized linearly, the radial mode of the Higgs potential, the twin sector Higgs, is present in the spectrum. This particle provides a new portal between the visible and twin sectors. We have shown that, if the discrete $Z_{2}$ symmetry is only softly broken, a measurement of the mass of the twin sector Higgs, when combined with precision measurements of the light Higgs, completely specifies the Higgs potential. The rates for twin sector Higgs events are then testable predictions of the twin Higgs framework. This conclusion also applies to theories that exhibit hard breaking of the $Z_{2}$ symmetry by the twin sector Yukawa couplings, provided that this breaking is small enough that the correction to the overall width of the twin sector Higgs is small. While the high luminosity LHC can potentially discover the twin sector Higgs, linear colliders such as the ILC or CLIC have much better precision and greater reach, allowing them to test the twin Higgs framework.

\section{ACKNOWLEDGMENTS}

We thank Jessie Shelton for helpful communication. S. N. would like to thank Aqeel Ahmed for useful discussions. Z. C. is supported in part by the National Science
Foundation under Grant No. PHY-1620074, the Fermilab Intensity Frontier Fellowship and the Visiting Scholars Grant No. 17-S-02 from the Universities Research Association. C. K. is supported by the National Science Foundation under Grant No. PHY-1620610. C. B. V. is supported by Department of Energy Grant No. DE-SC0009999. Z. C. would like to thank the Fermilab Theory Group for hospitality during the completion of this work. C.K. would also like to thank the Aspen Center for Physics, which is supported by National Science Foundation Grant No. PHY-1607611, where part of this work was completed.

\section{APPENDIX: HIGGS SECTOR COUPLINGS AND DECAYS}

This appendix includes several formulas relevant to the production and decays of Higgs bosons.

\section{Loop induced couplings of the Higgs fields}

Decays of the Higgs bosons to photons and gluons (we neglect the subdominant $Z \gamma$ channel) proceed through loops of electrically charged or colored particles. The expressions for these decay rates employ the functions

$$
\begin{gathered}
A_{F}(x)=2 x^{2}\left[\frac{1}{x}+\left(\frac{1}{x}-1\right) f(x)\right], \\
A_{V}(x)=-x^{2}\left[\frac{2}{x^{2}}+\frac{3}{x}+3\left(\frac{2}{x}-1\right) f(x)\right],
\end{gathered}
$$

with 


$$
f(x)= \begin{cases}\arcsin ^{2}\left(\frac{1}{\sqrt{x}}\right) & x \geq 1 \\ -\frac{1}{4}\left[\ln \left(\frac{1+\sqrt{1-x}}{1-\sqrt{1-x}}\right)-i \pi\right]^{2} & x<1\end{cases}
$$

The loop induced coupling of $h_{-}$to SM photons and gluons are reduced by a factor of $\cos (\vartheta-\theta)$, just like the tree level couplings. This is because each diagram involves only a single coupling of the Higgs to the particles running in the loop. This uniform reduction factors out of the overall amplitude.

The coupling of $h_{+}$to SM photons and gluons differs from the corresponding SM coupling in two ways. First, there is a uniform reduction of the tree level couplings by $\sin (\vartheta-\theta)$ that factors out of the amplitude, just as in the case of the light Higgs. Second, the loop functions $A_{F, V}(x)$ depend on the mass of the decaying particle through

$$
x=4 \frac{m_{F, V}^{2}}{m_{ \pm}^{2}}
$$

As the mass of the twin sector Higgs increases, $x$ decreases. Then the expressions for the decay width of the heavy Higgs to visible sector photons or gluons are the same as in the SM for a Higgs of mass $m_{+}$multiplied by $\sin ^{2}(\vartheta-\theta)$.

For instance, consider the decays of $h_{-}$and $h_{+}$to SM photons. To leading order, they are given by (see for instance [78])

$$
\begin{aligned}
& \Gamma\left(h_{-} \rightarrow \gamma_{A} \gamma_{A}\right)=\frac{\alpha_{A}^{2} m_{-}^{3}}{256 \pi^{3} v_{\mathrm{EW}}^{2}}\left|A_{V}\left(\frac{4 m_{W, A}^{2}}{m_{-}^{2}}\right)+\sum_{f_{A}} N_{c} Q_{f}^{2} A_{F}\left(\frac{4 m_{f, A}^{2}}{m_{-}^{2}}\right)\right|^{2} \cos ^{2}(\vartheta-\theta), \\
& \Gamma\left(h_{+} \rightarrow \gamma_{A} \gamma_{A}\right)=\frac{\alpha_{A}^{2} m_{+}^{3}}{256 \pi^{3} v_{\mathrm{EW}}^{2}}\left|A_{V}\left(\frac{4 m_{W, A}^{2}}{m_{+}^{2}}\right)+\sum_{f_{A}} N_{c} Q_{f}^{2} A_{F}\left(\frac{4 m_{f, A}^{2}}{m_{+}^{2}}\right)\right|^{2} \sin ^{2}(\vartheta-\theta),
\end{aligned}
$$

where $Q_{f}$ are the electric charges of the fermions and $N_{c}$ the number of colors of the various fermions. The corresponding expressions for decays to $B$-sector photons have exactly the same form, but now depend on the masses of the $B$ gauge bosons and fermions, while the $\cos (\vartheta-\theta)$ and $\sin (\vartheta-\theta)$ factors are exchanged. The form is illustrated by the leading order Higgs decays into $B$-sector gluons,

$$
\begin{gathered}
\Gamma\left(h_{-} \rightarrow g_{B} g_{B}\right)=\frac{\alpha_{s, B}^{2} m_{-}^{3}}{72 \pi^{3} v_{B}^{2}}\left|\frac{3}{4} \sum_{q_{B}} A_{F}\left(\frac{4 m_{q, B}^{2}}{m_{-}^{2}}\right)\right|^{2} \sin ^{2}(\vartheta-\theta), \\
\Gamma\left(h_{+} \rightarrow g_{B} g_{B}\right)=\frac{\alpha_{s, B}^{2} m_{+}^{3}}{72 \pi^{3} v_{B}^{2}}\left|\frac{3}{4} \sum_{q_{B}} A_{F}\left(\frac{4 m_{q, B}^{2}}{m_{+}^{2}}\right)\right|^{2} \cos ^{2}(\vartheta-\theta) .
\end{gathered}
$$

The production of Higgs bosons is similarly affected. The $h_{-}$production cross section is that of the SM but reduced by a factor of $\cos ^{2}(\vartheta-\theta)$, while the production cross section of $h_{+}$is that of a SM Higgs of mass $m_{+}$ reduced by a factor of $\sin ^{2}(\vartheta-\theta)$.

\section{Twin sector Higgs decays}

In this section we provide expressions for the widths of some of the important decay modes of the twin sector Higgs. The couplings of the $125 \mathrm{GeV}$ boson are constrained to be quite SM like. To be consistent with these bounds we require $v_{B} \gtrsim 3 v_{\mathrm{EW}}$. Then the twin sector Higgs is heavy enough to decay to real pairs of visible sector top quarks and gauge bosons. In our analysis, we make use of the formulas gathered in [78].

The partial width for twin sector Higgs decays into fermions is given by

$$
\Gamma\left(h_{+} \rightarrow f_{A, B} f_{A, B}\right)=\frac{N_{c} m_{+} Y_{f_{A, B}}^{2}}{16 \pi}\left(1-4 \frac{m_{f_{A, B}}^{2}}{m_{+}^{2}}\right)^{3 / 2}\left\{\begin{array}{cc}
\sin ^{2}(\vartheta-\theta) & f_{A} \\
\cos ^{2}(\vartheta-\theta) & f_{B}
\end{array} .\right.
$$

The corresponding expression for decays into on-shell pairs of $W$ and $Z$ gauge bosons is given by 


$$
\Gamma\left(h_{+} \rightarrow V_{A, B} V_{A, B}\right)=\frac{m_{+}^{3} \delta_{V}}{32 \pi} \sqrt{1-4 \frac{m_{V}^{2}}{m_{+}^{2}}}\left(1-4 \frac{m_{V}^{2}}{m_{+}^{2}}+12 \frac{m_{V}^{4}}{m_{+}^{4}}\right)\left\{\begin{array}{lc}
\frac{\sin ^{2}(\vartheta-\theta)}{v_{A}^{2}} & V_{A} \\
\frac{\cos ^{2}(\vartheta-\theta)}{v_{B}^{2}} & V_{B}
\end{array}\right.
$$

where $\delta_{W}=2$ and $\delta_{Z}=1$. If the twin sector Higgs is light, then decays to on-shell twin sector gauge bosons may be kinematically forbidden. In this case, one of the gauge bosons can be off shell, with the virtual particle decaying to lighter fermions, $h_{+} \rightarrow V V^{*} \rightarrow V f f$. In the limit that the final state fermions are massless the corresponding partial widths are given by

$$
\Gamma\left(h_{+} \rightarrow V_{B} V_{B}^{*}\right)=\frac{3 m_{+} m_{V}^{4} \delta_{V}^{\prime}}{32 \pi^{3} v_{B}^{4}} R_{T}\left(\frac{m_{V}^{2}}{m_{+}^{2}}\right) \cos ^{2}(\vartheta-\theta)
$$

where $\delta_{W}^{\prime}=1$ and

$$
\delta_{Z}^{\prime}=\frac{7}{12}-\frac{10}{9} \sin ^{2} \theta_{W}+\frac{40}{9} \sin ^{4} \theta_{W}
$$

Here

$$
R_{T}(x)=\frac{3\left(1-8 x+20 x^{2}\right)}{\sqrt{4 x-1}} \arccos \left(\frac{3 x-1}{2 x^{3 / 2}}\right)-\frac{1-x}{2 x}\left(2-13 x+47 x^{2}\right)-\frac{3}{2}\left(1-6 x+4 x^{2}\right) \ln x
$$

We now turn to twin sector Higgs decays into light Higgs bosons. Expanding out the Higgs potential, Eq. (7), we obtain a contribution to the cubic $h_{+} h_{-}^{2}$ coupling,

$$
\sigma^{3} \sqrt{2} f\left[\lambda+\delta-\frac{1}{2} \delta \sin ^{2}(2 \vartheta)\right]-\sigma^{2} h \frac{5 \delta f}{2 \sqrt{2}} \sin (4 \vartheta)-\sigma h^{2} \sqrt{2} \delta f\left[1-3 \sin ^{2}(2 \vartheta)\right]+h^{3} \frac{\delta f}{\sqrt{2}} \sin (4 \vartheta) .
$$

This leads to

$g_{h_{+} h_{-} h_{-}} \equiv \frac{2 f}{\sqrt{2}}\left\{3(\lambda+\delta) \sin \theta \sin (2 \theta)-\frac{\delta}{8}[\cos \theta-9 \cos (3 \theta)+2 \cos (\theta-4 \vartheta)+\cos (\theta+4 \vartheta)+21 \cos (3 \theta-4 \vartheta)]\right\}$.

There is also a contribution to the decay width from the kinetic term,

$$
\frac{1}{\sqrt{2} f} \sigma \partial_{\mu} h \partial^{\mu} h
$$

Then the partial width of $h_{+}$into $h_{-}$pairs is given by

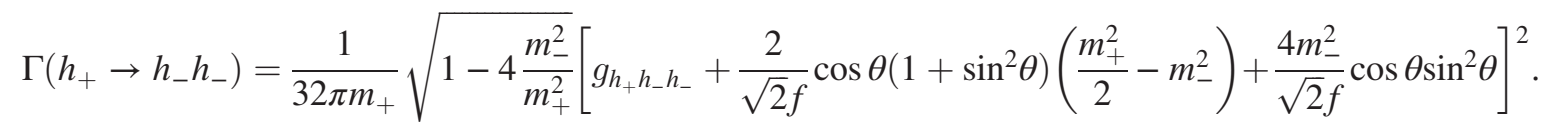

[1] G. Aad et al. (ATLAS Collaboration), Phys. Lett. B 716, 1 (2012).

[2] S. Chatrchyan et al. (CMS Collaboration), Phys. Lett. B 716, 30 (2012).

[3] P. Fayet, Phys. Lett. 69B, 489 (1977).

[4] S. Dimopoulos and H. Georgi, Nucl. Phys. B193, 150 (1981).
[5] N. Arkani-Hamed, A. G. Cohen, and H. Georgi, Phys. Lett. B 513, 232 (2001).

[6] Z. Chacko, H.-S. Goh, and R. Harnik, Phys. Rev. Lett. 96, 231802 (2006).

[7] R. Barbieri, T. Gregoire, and L. J. Hall, arXiv:hep-ph/ 0509242. 
[8] Z. Chacko, Y. Nomura, M. Papucci, and G. Perez, J. High Energy Phys. 01 (2006) 126.

[9] G. Burdman, Z. Chacko, H.-S. Goh, and R. Harnik, J. High Energy Phys. 02 (2007) 009.

[10] H. Cai, H.-C. Cheng, and J. Terning, J. High Energy Phys. 05 (2009) 045.

[11] D. Poland and J. Thaler, J. High Energy Phys. 11 (2008) 083.

[12] B. Batell and M. McCullough, Phys. Rev. D 92, 073018 (2015).

[13] J. Serra and R. Torre, Phys. Rev. D 97, 035017 (2018).

[14] C. Csáki, T. Ma, and J. Shu, arXiv:1709.08636.

[15] A. Falkowski, S. Pokorski, and M. Schmaltz, Phys. Rev. D 74, 035003 (2006).

[16] S. Chang, L. J. Hall, and N. Weiner, Phys. Rev. D 75, 035009 (2007).

[17] N. Craig and K. Howe, J. High Energy Phys. 03 (2014) 140.

[18] A. Katz, A. Mariotti, S. Pokorski, D. Redigolo, and R. Ziegler, J. High Energy Phys. 01 (2017) 142.

[19] M. Badziak and K. Harigaya, J. High Energy Phys. 06 (2017) 065.

[20] M. Badziak and K. Harigaya, J. High Energy Phys. 10 (2017) 109.

[21] M. Geller and O. Telem, Phys. Rev. Lett. 114, 191801 (2015).

[22] R. Barbieri, D. Greco, R. Rattazzi, and A. Wulzer, J. High Energy Phys. 08 (2015) 161.

[23] M. Low, A. Tesi, and L.-T. Wang, Phys. Rev. D 91, 095012 (2015).

[24] H.-C. Cheng, S. Jung, E. Salvioni, and Y. Tsai, J. High Energy Phys. 03 (2016) 074.

[25] H.-C. Cheng, E. Salvioni, and Y. Tsai, Phys. Rev. D 95, 115035 (2017).

[26] R. Contino, D. Greco, R. Mahbubani, R. Rattazzi, and R. Torre, Phys. Rev. D 96, 095036 (2017).

[27] C. Csaki, M. Geller, O. Telem, and A. Weiler, J. High Energy Phys. 09 (2016) 146.

[28] H. Beauchesne, K. Earl, and T. Grégoire, J. High Energy Phys. 01 (2016) 130.

[29] R. Harnik, K. Howe, and J. Kearney, J. High Energy Phys. 03 (2017) 111.

[30] J.-H. Yu, Phys. Rev. D 94, 111704 (2016).

[31] J.-H. Yu, J. High Energy Phys. 12 (2016) 143.

[32] J.-H. Yu, Phys. Rev. D 95, 095028 (2017).

[33] N. Craig, S. Knapen, and P. Longhi, Phys. Rev. Lett. 114, 061803 (2015).

[34] N. Craig, S. Knapen, and P. Longhi, J. High Energy Phys. 03 (2015) 106.

[35] N. Craig, S. Knapen, P. Longhi, and M. Strassler, J. High Energy Phys. 07 (2016) 002.

[36] K. Thrasher, arXiv:1705.01472.

[37] Z. Chacko, N. Craig, P. J. Fox, and R. Harnik, J. High Energy Phys. 07 (2017) 023.

[38] N. Craig, S. Koren, and T. Trott, J. High Energy Phys. 05 (2017) 038.

[39] M. Farina, J. Cosmol. Astropart. Phys. 11 (2015) 017.

[40] R. Barbieri, L. J. Hall, and K. Harigaya, J. High Energy Phys. 11 (2016) 172.
[41] C. Csaki, E. Kuflik, and S. Lombardo, Phys. Rev. D 96, 055013 (2017).

[42] R. Barbieri, L. J. Hall, and K. Harigaya, J. High Energy Phys. 10 (2017) 015.

[43] M. Farina, A. Monteux, and C. S. Shin, Phys. Rev. D 94, 035017 (2016).

[44] N. Craig, A. Katz, M. Strassler, and R. Sundrum, J. High Energy Phys. 07 (2015) 105.

[45] D. Curtin and C. B. Verhaaren, J. High Energy Phys. 12 (2015) 072.

[46] C. Csaki, E. Kuflik, S. Lombardo, and O. Slone, Phys. Rev. D 92, 073008 (2015).

[47] J. P. Chou, D. Curtin, and H. J. Lubatti, Phys. Lett. B 767, 29 (2017).

[48] D. Curtin and M.E. Peskin, Phys. Rev. D 97, 015006 (2018).

[49] N. Craig and A. Katz, J. Cosmol. Astropart. Phys. 10 (2015) 054.

[50] I. Garcia Garcia, R. Lasenby, and J. March-Russell, Phys. Rev. D 92, 055034 (2015).

[51] I. Garcia Garcia, R. Lasenby, and J. March-Russell, Phys. Rev. Lett. 115, 121801 (2015).

[52] M. Freytsis, S. Knapen, D. J. Robinson, and Y. Tsai, J. High Energy Phys. 05 (2016) 018.

[53] V. Prilepina and Y. Tsai, J. High Energy Phys. 09 (2017) 033.

[54] G. Burdman, Z. Chacko, R. Harnik, L. de Lima, and C. B. Verhaaren, Phys. Rev. D 91, 055007 (2015).

[55] R. Foot, H. Lew, and R. R. Volkas, Phys. Lett. B 272, 67 (1991).

[56] R. Foot, H. Lew, and R. R. Volkas, Mod. Phys. Lett. A 07, 2567 (1992).

[57] K. Fujii et al., arXiv:1506.05992.

[58] H. Abramowicz et al., Eur. Phys. J. C 77, 475 (2017).

[59] D. Buttazzo, F. Sala, and A. Tesi, J. High Energy Phys. 11 (2015) 158.

[60] A. Ahmed, J. High Energy Phys. 02 (2018) 048.

[61] T. Behnke, J. E. Brau, B. Foster, J. Fuster, M. Harrison, J. M. Paterson, M. Peskin, M. Stanitzki, N. Walker, and H. Yamamoto, arXiv:1306.6327.

[62] M. J. Boland et al. (CLICdp, CLIC Collaborations), arXiv: 1608.07537.

[63] G. Aad et al. (ATLAS, CMS Collaborations), J. High Energy Phys. 08 (2016) 045.

[64] S. Dawson et al., in Proceedings, 2013 Community Summer Study on the Future of U.S. Particle Physics: Snowmass on the Mississippi (CSS2013): Minneapolis, MN, USA (2013) arXiv: 1310.8361.

[65] ATLAS Collaboration, Report No. ATL-PHYS-PUB-2013016, 2013.

[66] CMS Collaboration, Report No. CMS-PAS-FTR-13-024, 2013.

[67] A. Holzner (ATLAS, CMS Collaborations), arXiv:1411 .0322 .

[68] D. de Florian et al. (LHC Higgs Cross Section Working Group), arXiv:1610.07922.

[69] LHC Higgs Cross Section Working Group, https://twiki .cern.ch/twiki/bin/view/LHCPhysics/LHCHXSWG, 2017. 
[70] Higgs cross sections for HL-LHC and HE-LHC, https://twiki .cern.ch/twiki/bin/view/LHCPhysics/HiggsEuropeanStrategy, 2016.

[71] M. L. Mangano et al., CERN, Yellow Report No. CERNTH-2016-112, 2017.

[72] J. Alwall, R. Frederix, S. Frixione, V. Hirschi, F. Maltoni, O. Mattelaer, H. S. Shao, T. Stelzer, P. Torrielli, and M. Zaro, J. High Energy Phys. 07 (2014) 079.

[73] T. Sjöstrand, S. Ask, J. R. Christiansen, R. Corke, N. Desai, P. Ilten, S. Mrenna, S. Prestel, C. O. Rasmussen, and P.Z. Skands, Comput. Phys. Commun. 191, 159 (2015).
[74] J. de Favereau, C. Delaere, P. Demin, A. Giammanco, V. Lemaître, A. Mertens, and M. Selvaggi (DELPHES 3 Collaboration), J. High Energy Phys. 02 (2014) 057.

[75] M. Cacciari, G. P. Salam, and G. Soyez, J. High Energy Phys. 04 (2008) 063.

[76] M. Cacciari, G. P. Salam, and G. Soyez, Eur. Phys. J. C 72, 1896 (2012).

[77] H. Abramowicz et al., arXiv:1306.6329.

[78] A. Djouadi, Phys. Rep. 457, 1 (2008). 